\title{
PROJECTIONS ON INVARIANT SUBSPACES ${ }^{1}$
}

\author{
WALTER RUDIN
}

1. Introduction. Let $L^{1}$ denote the Banach space of all Lebesgueintegrable functions on the unit circle, and let $H^{1}$ be the set of all $f \in L^{1}$ whose Fourier coefficients $\hat{f}(n)$ are 0 if $n<0$; here

$$
\hat{f}(n)=\frac{1}{2 \pi} \int_{-\pi}^{\pi} f(x) e^{-i n x} d x \quad(n=0, \pm 1, \pm 2, \cdots),
$$

and the arguments $x$ are understood to be real numbers modulo $2 \pi$.

It is clear that $H^{1}$ is a closed subspace of $L^{1}$. D. J. Newman has shown quite recently [3] that there exist no bounded projections of $L^{1}$ onto $H^{1}$. (By a projection we mean a linear operator $P$ such that $P^{2}=P$.) Certain facts about $H^{1}$ suggest a rather general setting for this type of theorem. The abstract problem to which one is thus led turns out to be quite easy, and, when specialized to $L^{1}$, yields a result which is much stronger than Newman's (Theorem 2).

Three facts about $H^{1}$ are relevant. First, $H^{1}$ is translation-invariant. That is to say, if the translation operators $\tau_{\nu}$ are defined by

$$
\left(\tau_{y} f\right)(x)=f(x-y)
$$

and if $f \in H^{1}$, then $\tau_{y} f \in H^{1}$.

Second, the "natural" projection

$$
\sum_{n=-\infty}^{\infty} c_{n} e^{i n x} \rightarrow \sum_{n=0}^{\infty} c_{n} e^{i n x}
$$

is known not to be bounded in the $L^{1}$-norm; there exist sequences $\left\{c_{n}\right\}$ such that the series on the left of (3) is the Fourier series of some $f \in L^{1}$, whereas the series on the right is not $[4$, p. 253].

Third, if $P$ were a projection of $L^{1}$ onto $H^{1}$ which commutes with all translation operators $\tau_{y}$, then $P$ would have to be of the form (3); this is easily verified by considering the action of $P$ on the characters $e^{i n x}$.

Thus there is no bounded projection of $L^{1}$ onto $H^{1}$ which commutes with all translations, although $H^{1}$ is translation-invariant. We shall see that this alone implies that there cannot exist any bounded projection of $L^{1}$ onto $H^{1}$.

Received by the editors March 30, 1961.

${ }^{1}$ Sponsored by the United States Army under Contract No. DA-11-022-ORD2059, Mathematics Research Center, United States Army, Madison, Wisconsin, and NSF Research Grant G-14362. 
2. For the general setting, let $X$ be a Banach space, let $G$ be a compact topological group, and suppose that $G$ acts as a group of bounded linear operators on $X$. This means: (a) There is an algebraic isomorphism $g \rightarrow T_{\theta}$ of $G$ onto a group of bounded linear operators $T_{\boldsymbol{\sigma}}$ on $X$ (they must be one-to-one and onto); we write $g x$ in place of $T_{g} x$. (b) The map $(g, x) \rightarrow g x$ is a continuous map of the product space $G \times X$ into $X$.

Theorem 1. If $X$ and $G$ are related in this fashion, if $Y$ is a closed subspace of $X$ which is invariant under $G$ (i.e., $g Y \subset Y$ for all $g \in G$ ), and if there exists a bounded projection $P$ of $X$ onto $Y$, then there exists $a$ bounded projection $Q$ of $X$ onto $Y$ which commutes with every $g \in G$.

Theorems of this type are standard tools in the theory of group representations, and this particular result may very well not be new.

Proof. For each positive integer $k$ and for each $x \in X$, the set $E_{k, x}$ consisting of all $g \in G$ for which $\|g x\| \leqq k\|x\|$ is closed. If $E_{k}$ is the set of all $g \in G$ for which $\|g\| \leqq k$, then $E_{k}$ is the intersection of all sets $E_{k, x}$ and is therefore also closed. Since $G$ is compact, the Baire theorem now implies that $\|g\|$ is bounded on some nonempty open set $V \subset G$, say $\|g\| \leqq B$ on $V$. If $g \in g_{0} V$, then $\|g\| \leqq B\left\|g_{0}\right\|$, and since $G$ is covered by finitely many translates of $V$, there is a number $M$ such that

$$
\|g\| \leqq M
$$$$
(g \in G) \text {. }
$$

We now define an operator $Q$ on $X$ by

$$
Q x=\int_{G} g^{-1} P g x d g \quad(x \in X) .
$$

Here $d g$ denotes the Haar measure of $G$, normalized so that the measure of $G$ is 1 . For every $x \in X, g^{-1} P g x$ is a continuous $X$-valued function on $G$. Hence $Q x$ is well defined by (5). It is evident that $Q$ is linear, and (4) shows that $\|Q\| \leqq M^{2}\|P\|<\infty$.

For any $x \in X, P g x \in Y$ for all $g \in G$, and since $Y$ is invariant under $G, g^{-1} P g x \in Y$. Since $Y$ is closed, it follows that $Q x \in Y$. Moreover, if $x \in Y$, then $g x \in Y, P g x=g x$, and so $g^{-1} P g x=x$; hence $Q x=x$. It follows that $Q$ is a bounded projection of $X$ onto $Y$.

Finally, fix $g_{0} \in G$ and put $h=g g_{0}$, so that $g^{-1}=g_{0} h^{-1}$. Since Haar measure is translation-invariant, we have

$$
Q g_{0} x=\int_{G} g^{-1} P g g_{0} x d g=\int_{G} g_{0} h^{-1} P h x d h=g_{0} Q x .
$$

This completes the proof. 
3. We now return to $L^{1}$. Let $S$ be a closed invariant subspace of $L^{1}$, i.e., if $f \in S$ then $\tau_{y} f \in S$ for all $y$, where $\tau_{y}$ is defined by (2). Since $S$ is closed, $S$ also contains the functions

$$
\hat{f}(n) e^{i n x}=\frac{1}{2 \pi} \int_{-\pi}^{\pi} f(x-y) e^{i n y} d y \quad(n=0, \pm 1, \pm 2, \cdots) .
$$

Thus, if $\hat{f}(n) \neq 0$ for some $f \in S$ and for some $n$, then $S$ contains the character $e^{i n x}$. Since every $f \in L^{1}$ can be approximated, in the $L^{1}$-norm, by finite linear combinations of partial sums of its Fourier series, we conclude that the closed translation-invariant subspaces of $L^{1}$ are determined by the characters they contain.

In other words, with every set $N \subset Z$ (where $Z$ is the set of all integers) there is associated a closed translation-invariant subspace $S_{N}$ of $L^{1}$, which consists of all $f \in L^{1}$ which have $\hat{f}(n)=0$ whenever $n$ is not in $N$; conversely, every closed translation-invariant subspace of $L^{1}$ is one of these spaces $S_{N}$.

If $Q$ is a bounded linear operator in $L^{1}$ which commutes with every $\tau_{y}$, then it is easily seen (by studying the action of $Q$ on a single character) that the Fourier coefficients of $Q f$ are of the form $c_{n} \hat{f}(n)$, where $\left\{c_{n}\right\}$ is a sequence determined by $Q$. Hence $\left\{c_{n}\right\}$ is a sequence of Fourier-Stieltjes coefficients $[4, \mathrm{p} .176]$. If, in addition, $Q$ is a projection onto $S_{N}$, then $c_{n}=1$ for $n \in N, c_{n}=0$ otherwise, i.e., $\left\{c_{n}\right\}$ is the characteristic function of $N$. But Helson has shown [2] that the only sets $N \subset Z$ whose characteristic functions are Fourier-Stieltjes transforms are the periodic sets (i.e., finite unions of arithmetic progressions, infinite in both directions), and those which differ from periodic sets in finitely many places.

The translation operators $\tau_{y}$ are isometries on $L^{1}$, and so

$$
\left\|\tau_{y} f-\tau_{y_{0}} f_{0}\right\|_{1} \leqq\left\|f-f_{0}\right\|_{1}+\left\|\tau_{y} f_{0}-\tau_{\nu_{0}} f_{0}\right\|_{1},
$$

which tends to 0 as $f \rightarrow f_{0}$ and $y \rightarrow y_{0}$. Hence the circle acts as a group of bounded linear transformations on $L^{1}$, and we can apply Theorem 1. The preceding remarks then immediately yield the following result:

TheOREM 2. Let $N$ be a subset of $Z$. There is a bounded projection of $L^{1}$ onto $S_{N}$ if and only if $N$ differs from a periodic set in at most finitely many places.

In particular, there is no such projection if $N$ is the set of all nonnegative integers; this is Newman's theorem.

4. In place of the integrable functions on the unit circle, we could 
equally well have discussed $L^{1}(G)$, the Banach space of all Haarintegrable functions on a compact abelian group $G$. We need only to know the idempotent measures on $G$, i.e., the measures whose FourierStieltjes coefficients are either 0 or 1 . Since the theorem of Helson, to which we referred in $\S 3$, has been extended to all compact abelian groups by Cohen [1], our discussion can be extended to this case without introducing any new ideas.

Theorem 1 can be applied to any function space on which the operators $\tau_{y}$ act continuously. A particularly simple example is furnished by the space $C$ of all continuous functions on the circle (or on any compact abelian group); the ideas of $\$ 3$ apply with very minor changes, and we shall merely state the result:

Theorem 2 holds with $C$ in place of $L^{1}$, if $S_{N}$ is interpreted to be the space of all $f \in C$ with $\hat{f}(n)=0$ outside $N$.

\section{REFERENCES}

1. P. J. Cohen, On a conjecture of Littlewood and idempotent measures, Amer. J. Math. 82 (1960), 191-212.

2. Henry Helson, Note on harmonic functions, Proc. Amer. Math. Soc. 4 (1953), 686-691.

3. D. J. Newman, The nonexistence of projections from $L^{1}$ to $H^{1}$, Proc. Amer. Math. Soc. 12 (1961), 98-99. 1959.

4. Antoni Zygmund, Trigonometric series, 2nd ed., Vol. I, Cambridge Univ. Press,

UNIVERSITY OF WISCONSIN 